\title{
COMPREENSÃO DE TEXTOS: CONSIDERAÇÕES SOBRE SEU DESENVOLVIMENTO
}

\section{TEXT COMPREHENSION: DEVELOPMENTAL CONSIDERATIONS}

\author{
Michelle França Dourado Neto Pires ${ }^{1}$ \\ Denise Dias Almeida ${ }^{2}$ \\ Universidade Federal de Pernambuco
}

\section{RESUMO}

A habilidade de compreender textos é essencial para a leitura, para a inserção do indivíduo na sociedade e para o sucesso escolar. Tendo em vista a importância que a compreensão de textos desempenha, o presente artigo tem como objetivo apresentar e refletir, a partir de uma revisão de literatura, como a compreensão de textos pode ser desenvolvida. Alguns autores demonstraram empiricamente que tal habilidade pode ser treinada a partir de tarefas especificas. Por fim, é proposta uma reflexão sobre como a escola pode proporcionar um contexto em que a compreensão de textos seja desenvolvida.

PALAVRAS-CHAVE: compreensão de textos; habilidade; desenvolvimento.

\begin{abstract}
The ability to understand texts is essential for reading, for the placement of the individual in society and school success. Given the importance reading comprehension plays, this article aims to present and reflect, from a literature review, how reading comprehension can be developed. Some authors have demonstrated empirically that this ability can be trained through specific tasks. Finally, the proposal is a reflection on how school can provide a context in which the text comprehension is developed.
\end{abstract}

KEYWORDS: text comprehension; ability; development.

\section{INTRODUÇÃO}

A compreensão de textos tem sido investigada por diversos pesquisadores (BAKER, 1996; FUCHS E FUCHS, 2007; HODGES E NOBRE, 2012; SPINILLO, 2008; SPINILLO E MAHON, 2007; YUILL E OAKHILL, 1991) com a finalidade de examinar quais processos estão envolvidos no ato de compreender o que se lê. A importância de estudar tal habilidade justifica-se pelo fato do sujeito estar inserido em uma sociedade letrada. Dessa maneira, grande parte das atividades que ele desempenha é envolvida pela prática da leitura (CORREA E MOUSINHO, 2013).

\footnotetext{
${ }^{1}$ Doutoranda no Programa de Pós-Graduação em Psicologia Cognitiva. E-mail: chelle_fd@hotmail.com

2 Doutoranda no Programa de Pós-Graduação em Psicologia Cognitiva. E-mail: denise_diasalmeida@hotmail.com
} 
Outrossim, a leitura é apontada como essencial no processo de aprendizagem escolar e como uma ferramenta que possibilita a transmissão dos aspectos culturais que constituem determinada sociedade (ROAZZI, HODGES, QUEIROGA, ASFORA E ROAZZI, 2013).

Cabe salientar que o elemento mais importante envolvido na leitura é a compreensão do que está sendo lido. Entende-se que a compreensão de textos é um processo no qual significados são construídos a partir da junção entre dois aspectos, sendo estes: as informações que estão contidas no texto (informações literais) e as inferências (SPINILLO, 2008).

Em concordância com essa ideia, Vidal-Abarca e Rico (2003) afirmam que para compreender um texto adequadamente é necessário que o leitor entenda as informações que estão escritas explicitamente e aquelas que são trazidas pelo leitor a partir de suas experiências, as quais estão armazenadas na sua memória.

Dessa maneira, a compreensão de textos não consiste simplesmente na decodificação das palavras de um texto de maneira correta, mas vai muito além disso. Compreender é uma atividade integrativa e construtiva, na qual o leitor tem acesso às informações contidas nos textos e tenta de maneira ativa preencher as lacunas deixadas pelo autor através do seu conhecimento de mundo, construindo ao final da leitura uma representação mental significativa do texto (SPINILLO, 2008).

Nesse sentido, Spinillo (2013) aponta que a compreensão de textos seria envolvida por três instâncias: o leitor, o texto e a interação entre eles. Dentro dessa interação três dimensões estariam entrelaçadas, sendo elas: a social, a linguística e a cognitiva. Em relação à dimensão social, destaca-se o conhecimento de mundo, que é construído pelas próprias experiências sociais do sujeito, podendo ser elas formais e informais.

$\mathrm{Na}$ dimensão linguística, ressaltam-se as capacidades de decodificação e de reconhecimento, como aspectos importantes para a compreensão de textos. Quando ocorrem problemas relacionados à decodificação pode acontecer uma sobrecarga na memória de trabalho, dificultando a integração das informações contidas no texto, e por consequência a compreensão. É importante salientar que a decodificação é uma capacidade necessária, mas que apenas ela não garante a compreensão, posto que outros aspectos, dentre eles as inferências, estão envolvidos nesse processo (SPINILLO, 2013).

No que concerne à dimensão cognitiva, destaca-se: a memória, o monitoramento, as inferências e as imagens mentais. A memória de trabalho e de longo prazo atuam na compreensão de maneira distinta, a medida que a primeira está relacionada a habilidade do leitor de reter as informações textuais em um curto período, torna possível a identificação de inconsistências contidas no texto. A segunda estaria relacionada às experiências do sujeito que ficam armazenadas durante longo período (SPINILLO, 2013).

O monitoramento se refere à capacidade do sujeito em ter consciência do seu nível de compreensão do texto, sendo capaz de identificar suas dúvidas e dificuldades e a partir disso poder traçar estratégias para melhorar sua compreensão (SPINILLO, 2013).

As inferências dizem respeito à capacidade do sujeito em preencher as lacunas presentes no texto, já que nem tudo está escrito de maneira explicita. O estabelecimento das inferências pode ocorrer de duas maneiras, a primeira seria a partir da integração das informações que estão contidas no texto, envolvendo conexões entre as ideias. A segunda seria a partir da ligação entre as passagens do texto e o conhecimento prévio do leitor (SPINILLO E HODGES, 2012; VIDAL-ABARCA E RICO, 2003).

Assim sendo, diversos fatores estão envolvidos no processo de compreensão de texto. Além do mais, cabe salientar a importância que a compreensão desempenha para o sucesso escolar, para lidar com situações cotidianas, tendo em vista que grande parte delas envolve a leitura. Dessa maneira, pesquisas que busquem desenvolver essa habilidade são de grande importância. 
Sendo assim, este trabalho tem por objetivo apresentar e refletir sobre os estudos que tiveram o intuito de desenvolver a habilidade de compreensão de texto. Além disso, serão discutidas como as diferentes habilidades cognitivas podem influenciar, de maneira distinta, o desenvolvimento da compreensão de textos. Para tanto, serão expostos os estudos de intervenção e aqueles que tiveram o objetivo de promover o desenvolvimento de tal habilidade.

\section{Pesquisas sobre compreensão de textos}

Brandão e Spinillo (1998) alertam que nem toda tarefa de compreensão consegue apontar os diferentes fatores envolvidos neste processo. Geralmente investiga-se a compreensão com tarefas de reprodução, seja oral ou escrita, de um texto (lido, ouvido ou apresentado em gravuras). Também é comum a realização de perguntas sobre um determinado texto. Quando as crianças são muito pequenas aplicam-se a reprodução de texto na forma oral. Já as tarefas de perguntas são realizadas com sujeitos com habilidade de leitura, com mais tempo de escolarização.

A pesquisa de Spinillo e Mahon (2007) utilizou as tarefas de perguntas. As autoras tinham como objetivo investigar se a capacidade de crianças em estabelecer inferências durante a leitura de um texto varia em função da natureza da informação inferencial, e se essa capacidade se altera em função da idade/escolaridade. Quarenta crianças estudantes da $1^{\text {a }}$ (idade média de 7 anos e 2 meses) e $3^{a}$ série ( 9 anos e 4 meses) do ensino fundamental responderam a perguntas inferenciais (causal, de estado e previsão) sobre um texto narrativo.

Os resultados mostraram que em relação à pergunta causal houve uma tendência de responder de forma coerente ou provável nos dois grupos. Nas perguntas de estado ocorreram diferenças em perguntas específicas de acordo com a idade, como a que envolvia informações referentes a espaço. As crianças de sete anos apresentaram mais dificuldades nas perguntas temporais. As crianças mais novas tendiam a não responder às perguntas de previsão. As autoras observaram que os estudantes foram capazes de estabelecer as inferências, e que as respostas coerentes foram mais frequentes nas crianças mais velhas.

Observa-se na literatura pesquisas que utilizam duas tarefas, como por exemplo, de decodificação em conjunto com as de compreensão. É o caso do estudo de Spinillo e Hodges (2012) que analisaram e identificaram os erros de crianças com dificuldade de compreensão e sua relação com a elaboração de inferências. Examinaram se os erros variavam em relação à metodologia de leitura on-line e off-line. Como hipótese, as autoras consideravam que a leitura online (interrompida) diminuiria o número e a frequência de algum tipo específico de erro.

Desta maneira, selecionaram quarenta participantes que não demonstraram problemas de decodificação, entretanto apresentaram problemas na elaboração de inferências. Cada participante foi entrevistado individualmente em duas sessões: na primeira foram aplicadas as tarefas de sondagem da decodificação (leitura em voz alta de um texto) e de compreensão (leitura em voz alta de duas histórias seguida de perguntas literais e inferenciais). $\mathrm{Na}$ segunda sessão dividiram os participantes com dificuldade de leitura em dois grupos considerando a idade, a escolaridade e o sexo.

Observaram que os tipos de erros revelaram diferentes níveis de dificuldades, dos mais elementares (trata as informações de forma isolada) aos mais elaborados (indicam a elaboração das inferências que distorcem ou extrapolam). Os resultados permitiu identificar três diferentes tipos de erros quanto o estabelecimento de inferências pelas crianças: do tipo 2 em que considera o texto, mas não integra as informações intratextuais; o tipo 3 que considera o texto, há a tentativa de integrar as informações intratextuais, mas de forma desautorizada; e o tipo 4 em que consideram o texto e os conhecimentos prévios como tentativa de integrar as informações intratextuais, mas de forma desautorizada pelo texto. 
A metodologia de leitura pareceu não influenciar na frequência de acertos e erros, o que indica que as dificuldades são estáveis. Apesar disso, ao analisar os tipos de erros identificou-se diferenças entre as duas metodologias de leitura: na forma on-line observou-se a integração de informações intra e extratextuais, posto que os erros tipo 4 fossem mais frequentes.

Nos estudos de compreensão de textos com crianças é corriqueiro o uso de texto narrativo, com poucos estudos sobre a compreensão em diferentes tipos textuais. Com o objetivo de estudar as relações entre compreensão e tipos de textos, Spinillo e Almeida (2014) utilizaram textos narrativo (história) e argumentativo (texto de opinião). Investigaram ainda se as características estruturais dos dois textos influenciariam a compreensão do leitor. Utilizou-se a metodologia on-line de leitura, pois esta divisão em blocos possibilita realizar perguntas inferenciais de previsão, além das causais e de estado. Participaram do estudo 100 crianças com idade de 8 e 9 anos, de classe média, alunos do $3^{\circ}$ e $4^{\circ}$ anos do ensino fundamental de escolas particulares. Cada criança foi entrevistada individualmente em duas sessões, em que cada sessão era utilizada um tipo de texto seguido da realização das perguntas de ordem fixa e das de explicação (metacognitivas).

De acordo com os resultados, o tipo de inferência parece influenciar o tipo de resposta dada pela criança, visto que as inferências causais e de estado foram mais fáceis de serem estabelecidas dos que as inferências de previsão. Nos dois tipos de texto as crianças tendiam a ter um bom nível de compreensão. No texto narrativo, tendiam a fornecer respostas apropriadas e completas, e no texto argumentativo as crianças tendiam a dar respostas apropriadas, porém incompletas. Ressalta-se que as relações inferenciais de causalidade, estado ou previsão variaram em função do tipo de texto que está sendo compreendido, assumindo características relacionadas às propriedades do texto.

Os estudos citados contribuem para a investigação dos processos envolvidos na compreensão ao utilizarem tarefas diversificadas como teste de sondagem da decodificação, perguntas inferenciais de diferentes tipos, perguntas de explicação. $O$ uso de diferentes metodologias de leitura (on-line e off-line) e os diferentes tipos de texto contribui na tentativa de investigação da habilidade de compreender textos.

\section{Compreensão de textos: como desenvolver}

O processo de ler um texto e compreendê-lo envolve diversos aspectos, podendo ser sociais, linguísticos e cognitivos. Nesse sentido, existem diversos fatores responsáveis por uma boa compreensão textual (SPINILLO, 2013).

Os estudos que têm o objetivo de desenvolver a habilidade de compreensão de textos utilizam tarefas que buscam melhorar e aumentar as habilidades que estão relacionadas com esse processo. Dentre essas habilidades pode-se destacar: o uso de imagens mentais, inferências, memória de trabalho, monitoramento, entre outros.

Buscando observar como o treinamento de inferências pode influenciar o nível de compreensão, Yuill e Oakhill (1991) realizaram um experimento que objetivava habilitar as crianças a fazer inferências. Os sujeitos foram divididos em grupo experimental e grupo controle, os dois grupos eram constituídos por crianças com e sem dificuldade na compreensão.

Foram utilizadas oito histórias experimentais e duas histórias práticas com um estilo altamente abstrato, deixando implícita uma série de informações. As crianças do grupo experimental recebiam um treinamento que as orientava a prestar atenção em algumas palavras que serviam como pistas para compreender o texto.

Além disso, em outro momento, as crianças tinham acesso a um texto diferente, e era solicitado que identificassem as palavras que serviam como pistas para responder as perguntas. $\mathrm{O}$ examinador comentava o seu desempenho, e indicava as pistas que não foram citadas pelas 
crianças. Por outro lado, as crianças que pertenciam ao grupo controle não recebiam nenhum treinamento ou direcionamento com as palavras chaves.

Os resultados demonstraram um efeito significativo do treinamento em relação ao nível de compreensão daquelas crianças menos habilidosas, sendo verificado que a intervenção auxiliou as crianças a focarem nas partes mais importantes do texto.

Ademais, as crianças com dificuldades em compreensão que foram treinadas apresentaram um desempenho significativamente melhor do que as do grupo controle. E atingiram um nível de compreensão semelhante aos que tinham um bom nível de compreensão.

Esses dados indicam que o uso de instruções e a indicação de pistas durante a leitura podem ajudar as crianças com dificuldades de compreensão textual. Os resultados ainda sugerem que as instruções melhoram as habilidades inferenciais, e por consequência, a compreensão de texto.

Em outro estudo, além de verificar o efeito do treinamento de inferências, Yuill e Oakhill (1991) buscaram averiguar como o desenvolvimento do monitoramento influencia a habilidade de compreender textos. As crianças foram divididas do mesmo modo que no estudo descrito anteriormente. $\mathrm{O}$ treinamento para o desenvolvimento da capacidade inferencial também foi o mesmo.

Em relação ao treinamento do monitoramento, as crianças foram introduzidas à ideia das "palavras interrogativas", sendo elas: quem, onde, por quê, e quando. Foi explicado como essas palavras poderiam auxiliar a formular perguntas sobre o texto. Esse procedimento tinha o objetivo de aumentar a consciência das crianças sobre a sua compreensão textual e ajudar a elaborar perguntas que conduziriam a sua compreensão. Essa intervenção também encorajava a criança a fazer inferências preditivas sobre o que estavam lendo.

Os resultados apontaram que as crianças que tinham um baixo nível de compreensão foram mais beneficiadas pelo treinamento com o uso de inferências, quando comparadas com as crianças que já tinham um alto nível de compreensão. Além do que, os dados demonstraram que o treinamento que visava o aumento do monitoramento, teve um efeito semelhante ao treinamento com o uso de inferências.

Esses achados sugerem que o uso de perguntas, durante a leitura, direciona as crianças a focarem nas partes mais importantes do texto. Essa estratégia possibilita que a criança infira informações que não estão presentes no texto, ou que ainda não foram fornecidas.

Yuill e Oakhill (1991) desenvolveram uma pesquisa com o objetivo de verificar quais aspectos do treinamento com o uso de inferências e do treinamento com monitoramento influenciam na compreensão. Neste estudo existiam dois grupos experimentais e um grupo controle.

No grupo de treinamento inferencial, as crianças tiveram acesso à prática para encontrar as palavras chaves no texto e tentativas de adivinhar sentenças implícitas. No grupo de monitoramento da compreensão, as crianças receberam cartões com "palavras perguntas", tais como: por quê, o quê, quando. Os sujeitos foram ensinados a construir questões sobre o texto utilizando essas palavras. O teste Neale foi utilizado para verificar o efeito do treinamento, sendo aplicado no pré e pós-teste, uma semana após o fim do treinamento e seis meses depois.

Os resultados demonstraram que o monitoramento parece ser mais efetivo quando combinado com o treino inferencial. Dessa maneira, os dois tipos de treinamento auxiliam no desenvolvimento da compreensão, visto que ambos fornecem ferramentas para que a criança se torne ativa durante o processo da leitura.

Em concordância com a suposição de que a habilidade de monitoramento estaria relacionada com uma boa compreensão, Coelho e Correa (2010) realizaram seu estudo com adolescentes, tendo como objetivo desenvolver a habilidade de monitoramento através do emprego do paradigma de detecção de erros. 
Os participantes foram divididos em grupo experimental e controle. Foi utilizada a tarefa de Cloze para avaliar a compreensão antes e depois do treinamento, sendo, portanto, aplicada no pré e pós-teste. O monitoramento foi medido a partir do paradigma da detecção dos erros, no qual são feitas inserções de transgressões no texto, tanto no âmbito lexical como de consistência interna, e o sujeito deveria identificar e corrigir os erros.

Os resultados demonstraram que no pós-teste os participantes que pertenciam ao grupo experimental melhoraram o seu desempenho no monitoramento e na compreensão de textos, quando comparadas com os que faziam parte do grupo controle. Foi verificado que os leitores menos habilidosos, que pertenciam ao grupo experimental, tiveram um melhor desempenho no pós-teste quando comparados com aqueles que pertenciam ao grupo controle.

Nesse sentido, esse estudo evidenciou que as habilidades metacognitivas, nesse caso o monitoramento, estão relacionadas com uma boa compreensão de textos. Ainda foi possível verificar que o uso de um treinamento adequado possibilitou o desenvolvimento de tais habilidades, pois os adolescentes se tornaram conscientes de possíveis erros presentes no texto e a partir disso puderam traçar estratégias para corrigi-los (COELHO E CORREA, 2010).

Outros estudos buscaram verificar o efeito das imagens mentais sobre a compreensão de textos. Yuill e Oakhill (1991) realizaram sua pesquisa com o intuito de examinar se o uso de imagens mentais auxilia na memória de alguns tipos de informações advindos do texto, e desse modo capacitar a criança a obter uma representação mental adequada do texto.

As crianças foram divididas em grupo controle e experimental, neste último recebia o treinamento com o uso de imagens mentais. Os grupos eram constituídos por participantes com baixos e altos níveis de compreensão. Foram utilizadas nove histórias com vocabulário adequado, desse modo, todos os sujeitos poderiam ler sem nenhuma dificuldade. Quatro histórias foram utilizadas nas sessões de treinamento e cinco na sessão de teste. Após cada passagem, foram feitas três tipos de questões para as crianças, sendo elas: inferencial, descritiva e factual.

O treinamento foi dividido em três sessões e foi dito aos sujeitos que eles iriam aprender a pensar em forma de imagens. No primeiro treino, as crianças leram uma história e o experimentador mostrou dois desenhos: sequência de quatro figuras que representavam a continuação de eventos da história, e uma figura única que descrevia o evento principal da história.

Após esse momento, as crianças foram solicitadas a fazer uma imagem mental a respeito do que tinham lido e a formular um desenho do que elas achavam ser o principal evento. Em seguida o experimentador solicitava que descrevessem as suas imagens mentais, e a partir de seus relatos era dado um feedback de como essas imagens poderiam ser aprimoradas.

$\mathrm{Na}$ segunda sessão de treinamento foi dito para as crianças que elas deveriam tentar formar três tipos de imagens diferentes: um desenho com quatro figuras, uma imagem do evento principal e uma imagem que envolvia o nome do personagem principal. As imagens feitas pelas crianças foram discutidas com o experimentador.

$\mathrm{Na}$ terceira sessão não foi mostrado nenhum desenho para as crianças. Foi utilizado apenas o procedimento para formular imagens mentais, e após isso suas imagens foram discutidas com o experimentador.

Os resultados demonstraram que os participantes menos habilidosos, os quais receberam o treinamento, apresentaram melhora ao responder as questões. Esses dados sugerem que o treinamento com imagens mentais é eficaz para aperfeiçoar o desempenho das crianças que possuem um baixo nível de compreensão. Mais especificamente, no que se refere às questões que requerem habilidades inferenciais ou informações de natureza descritiva, mas que não estão explícitas no texto. Além disso, as imagens mentais podem ter auxiliado na formulação de uma representação mental adequada do texto, o que permitiria que as crianças utilizassem a sua memória de trabalho de maneira mais eficaz. 
Buscando também verificar os efeitos das imagens mentais e de tomar notas sobre a compreensão de textos, Ferreira e Dias (2002) desenvolveram um estudo com crianças entre oito e quatorze anos de escolas públicas e particulares. Os sujeitos foram classificados nos grupos por meio da dificuldade de compreensão apresentada (pouco ou muita), sendo distribuídos em dois grupos experimentais e um grupo controle.

No treinamento de tomar notas, os participantes foram instruídos a tomar notas, com suas próprias palavras, das passagens do texto que achassem importantes. E foram alertados de que essa estratégia era uma boa maneira de obter a compreensão do que estava sendo lido.

No treinamento de imagem mental, da mesma maneira que no estudo de Yuill e Oakhill (1991), as crianças foram instruídas a fazer "desenhos na sua cabeça" em cada sequência lida do texto. Sendo alertadas de que essa é uma boa maneira de se lembrar da história que foi lida.

Os resultados apontaram que as crianças, as quais receberam o treinamento de tomar notas tiveram um desempenho melhor no pós-teste, quando comparadas com aquelas que foram submetidas ao treinamento com o uso de imagens mentais, e aquelas que pertenciam ao grupo controle.

Esses dados sugerem uma maior eficácia da estratégia de tomar notas para o desenvolvimento da habilidade de compreensão, quando comparada com a imagem mental. No entanto, vale salientar que ambas as estratégias são eficazes para auxiliarem na construção de inferências, e consequentemente promover a compreensão de textos.

Dias, Ferreira e Roazzi (2010) consideram que o treinamento com o uso de imagens mentais seria uma importante ferramenta para desenvolver a compreensão textual. Segundo esses autores, tal estratégia é fácil de ser ensinada e utilizada, pois não necessita de um treinamento longo. Além de ter baixo custo, pois faz uso apenas de palavras, sem o uso de materiais adicionais como slides ou filmes. Pontuam ainda a necessidade do leitor ter participação ativa no processo de leitura e de compreensão, o que possibilita o processo de aprendizagem das estratégias de leitura.

Os estudos citados anteriormente apontam para uma série de habilidades e estratégias que quando treinadas adequadamente, podem auxiliar no desenvolvimento da compreensão de textos. No entanto, nenhuma pesquisa teve o objetivo de observar como esse treinamento pode ocorrer de maneira prática, em sala de aula, com o auxílio dos professores.

Partindo da possibilidade de promover a compreensão com o auxílio dos educadores, Spinillo (2008) realizou um estudo de intervenção em sala de aula, o qual envolvia o treinamento com a professora responsável. Para tanto, essa autora buscou desenvolver a compreensão de textos, em crianças com dificuldades, fazendo uso de um treinamento com o estabelecimento de inferências.

Após um pré-teste, as crianças com mesmo nível de dificuldade foram distribuídas no grupo experimental e no grupo controle. No grupo experimental, a intervenção foi conduzida pela professora na própria sala de aula. Vale salientar que a docente foi orientada, antes e durante a intervenção, pela pesquisadora. A intervenção consistia em atividades individuais e em grupo com a leitura de textos de diferentes tipos: cartas, poesias, textos jornalísticos, entre outros.

A professora conduzia os alunos, durante a leitura, a realizarem as seguintes tarefas: estabelecer inferências a partir das informações textuais e fora dele (conhecimento de mundo), extrair as informações principais do texto, monitorar a leitura, e explicitar o que levou a chegar a determinada resposta ou ter certo julgamento sobre o texto.

A leitura dos textos foi realizada de duas maneiras: I) metodologia off-line, a qual consiste em fazer perguntas sobre o texto apenas ao final da leitura; II) metodologia on-line, que se caracteriza pelo fato de ser uma leitura interrompida, pois são feitas perguntas após cada passagem lida.

Os resultados demonstraram que a intervenção teve um efeito positivo sobre a compreensão. Sendo também observada a ocorrência de avanço na capacidade da criança em 
estabelecer e explicar o que gerou as inferências. Esses dados sugerem que o treinamento em sala de aula com o uso de atividades inferenciais e de monitoramento proporciona o desenvolvimento de maiores níveis de compreensão para as crianças.

Recentemente, Miragaia e Balula (2017) desenvolveram um estudo com o objetivo de melhorar a compreensão da leitura através do ensino explicito de estratégias de leitura, dentre elas, o uso de inferências. O estudo foi dividido em duas etapas, na primeira foi avaliado o desempenho em atividades de compreensão de leitura de 111 crianças da $5^{a}$ série, com idades abrangidas entre nove aos doze anos, para tanto foi realizada a aplicação de uma prova de leitura . $\mathrm{Na}$ segunda etapa, foi realizada uma intervenção em contexto de sala de aula com 33 alunos da $5^{\mathrm{a}}$ série. Esta intervenção era destinada ao ensino explicito de estratégias de leitura, as quais visavam a melhoria da compreensão.

Os resultados apontaram para uma melhoria nos domínios: compreensão inferencial, na reorganização e compreensão crítica após a realização da intervenção. Apontando que o ensino com o uso de inferências é uma possibilidade que auxilia na melhoria da compreensão da leitura.

A partir dos estudos citados, pode-se constatar que é possível desenvolver a habilidade de compreensão de textos. Para tanto, é necessário que os fatores cognitivos (inferência, metacognição, imagens mentais) os quais estão envolvidos no processo de compreensão sejam treinados de maneira eficaz.

\section{CONSIDERAÇÕES FINAIS}

O presente trabalho objetivou verificar e discutir os estudos que buscam desenvolver a habilidade de compreensão de textos. Foi observado que esse desenvolvimento é possível a partir do treinamento de aspectos que estão relacionados com o processo de compreensão.

Mesmo possuindo o conhecimento de que a habilidade de compreender texto é possível de ser desenvolvida, a maioria dos educadores não tem uma prática educacional voltada para o desenvolvimento de tal habilidade. Spinillo (2013) aponta para duas possíveis razões que levariam os professores a terem esse comportamento.

A primeira razão seria a crença que a habilidade de compreender texto seria desenvolvida durante os anos de escolarização. Desse modo, supõe-se que a criança ao ter o domínio sobre o sistema de representação da escrita, teria também a capacidade de compreender o que está sendo lido. A segunda, de que os livros didáticos seriam suficientes para desenvolver essa habilidade. No entanto, essas suposições não foram corroboradas (BUARQUE, HIGINO, MIRANDA, DUBEUX E PEDROSA, 1992; MARCUSCHI, 1996). Foi encontrado que a maioria das atividades dos livros se baseia na copiação, e que atividades de reflexão são raramente solicitadas.

Levando em consideração os achados dos estudos mencionados, faz-se necessário repensar as práticas escolares e os materiais didáticos utilizados dentro da sala de aula, visto que esses não têm contribuído para o desenvolvimento da compreensão de texto. Dessa maneira, devem-se propor ao educador novas atividades de ensino que abarque o treinamento de estratégias que estão envolvidas na compreensão.

Entre as atividades que poderiam ser utilizadas em sala de aula, Spinillo (2013) sugere as seguintes: o uso de imagens mentais durante a leitura de cada sentença do texto; solicitar que indiquem as palavras que auxiliaram a chegar à determinada conclusão sobre o texto; monitorar a sua compreensão; elaborar perguntas; estabelecer e explicitar inferências.

Esse treinamento é importante posto que algumas crianças menos habilidosas necessitam ser ensinadas a usar essas habilidades. Como foi observado nos estudos realizados por Yuill e Oakhill (1991), nos quais as crianças que eram consideradas más compreendedoras necessitavam de treinamentos específicos para alcançarem níveis mais avançados na habilidade de compreender textos. 
Diante do que foi exposto, pode-se observar que a habilidade de compreender textos deve ser considerada, pelos educadores, como um objeto de ensino. Visto que, como aponta Marcuschi (1996), essa habilidade não é algo inato e nem transmitida geneticamente, portanto, deve ser treinada. Dessa maneira, deve-se fazer uso de tarefas que proporcionem o uso das estratégias que auxiliam o desenvolvimento da compreensão.

\section{REFERÊNCIAS BIBLIOGRÁFICAS}

BAKER, Linda. Social influences on metacognitive development in reading. In CORNOLDI, C; OAKHILL, J. (Eds.). Reading comprehension difficulties: Processes and intervention. Mahwah, NJ: Lawrence Erlbaum Associates, p.331-352, 1996.

BRANDÃO, Ana Carolina Perrusi; SPINILLO, Alina Galvão. Aspectos gerais e específicos na compreensão de textos. Psicologia Reflexão e Crítica. 11 (2), pp. 253-272, 1998.

BUARQUE, Lair L; HIGINO, Zélia M; MIRANDA, Elisabete M; DUBEUX, Maria Helena; PEDROSA, Ivani. Avaliação do desempenho da rede pública escolar do Estado de Pernambuco na área de linguagem. Estudos em Avaliação Educacional, v. 5, p.95-106, 1992.

COELHO, Carmen Lúcia Göbel; CORREA, Jane. Desenvolvimento da Compreensão Leitora através do Monitoramento da Leitura. Psicologia: Reflexão e Crítica, v.23, n.3, p.575-581, 2010.

CORREA, Jane; MOUSINHO, Renata. Por um modelo simples de leitura, porém não tão simples assim. In MOTA, M; SPINILLO, A. G (Orgs.), Compreensão de textos: processos e modelos. São Paulo: Casa do Psicólogo, p.77-100, 2013.

DIAS, Maria da Graça Bompastor Borges; FERREIRA, Sandra Patrícia Ataíde; ROAZZI, Antonio. Exercícios e estratégias aplicados em sala de aula para uma melhor compreensão de texto. In GUIMARÃES, S. R. K; MALUF, M. R. (Orgs). Aprendiragem da linguagem escrita. São Paulo: Vetor, p.343-354, 2010.

FERREIRA, Sandra Patrícia Ataíde; DIAS, Maria da Graça Bompastor Borges. Dificuldades de compreensão: estratégias de tomar notas e da imagem mental. Psicologia: Teoria e Pesquisa. v.18, n.1, p. $51-62,2002$.

FUCHS, Doug; FUCHS, Lynn. Increasing Strategic Reading Comprehension with Peer-Assisted Learning Activities. In MCNAMARA, D. S. (Org.) Reading Comprehension Strategies: Theories, Interventions, and Technologies. Nova York: Lawrence Erlbaum Associates, p.175-197, 2007.

HODGES, Luciana Vasconcelos dos Santos Dantas; NOBRE, Alena Pimentel Mello Cabral. O uso de estratégias metacognitivas como suporte à compreensão textual. Revista Eletrônica de Educação, v. 6, n.2, p.476-490, 2002.

MARCUSCHI, Luis Antonio. Exercícios de compreensão ou copiação nos manuais de ensino de língua? Aberto, Brasília, v.16, n.69, p.64-82, 1996.

MIRAGAIA, Etelvina-i-j; BALULA, João Pauloł. Estratégias para o desenvolvimento da compreensão de textos literários na sala de aula. In MENEZES, L; CARDOSO, A. P; REGO, B; 
BALULA, J. P; FIGUEIREDO, M; FELIZARDO, S. (Eds). Olhares sobre a Educação: em torno da formação de professores-. Viseu: Escola Superior de Educação de Viseu (ESEV), p. 131-139, 2017.

ROAZZI, Antonio; HODGES, Luciana Vasconcelos dos Santos Dantas; QUEIROGA, Bianca; ASFORA, Rafaella; ROAZZI, Maira Monteiro. Compreensão de texto e modelos teóricos explicativos: a influência de fatores linguísticos, cognitivos e metacognitivos. In MOTA, M; SPINILLO, A. G. (Orgs.), Compreensão de textos: processos e modelos. São Paulo: Casa do Psicólogo, p.41-76, 2013.

SPINILLO, Alina Galvão. O leitor e o texto: desenvolvendo a compreensão de textos na sala de aula. Interamerican Journal of Psychology, v.42, n.1, p.29-40, 2008.

SPINILLO, Alina Galvão. A dimensão social, linguística e cognitiva da compreensão de textos: considerações teóricas e aplicadas. In MOTA, M; SPINILLO, A. G. (Orgs.), Compreensão de textos: processos e modelos. São Paulo: Casa do Psicólogo, p.171-198, 2013.

SPINILLO, Alina Galvão; ALMEIDA, Denise Dias. Compreendendo textos narrativo e argumentativo: há diferenças? Arquivos Brasileiros de Psicologia. Rio de Janeiro, 66(3), pp. 115-132, 2014.

SPINILLO, Alina Galvão; HODGES, Luciana Vasconcelos dos Santos Dantas. Análise de erros e compreensão de textos: comparações entre diferentes situações de leitura. Psicologia: Teoria e Pesquisa, v.28, n.4, p.381-288, 2012.

SPINILLO, Alina Galvão; MAHON, Erika da Rocha. Compreensão de Texto em Crianças: Comparações entre Diferentes Classes de Inferência a partir de uma Metodologia On-line. Psicologia: Reflexão e Crítica, v.20, n.3, p.463-471, 2007.

VIDAL-ABARCA, Eduardo; RICO, Gabriel Martínez. Por que os textos são tão difíceis de compreender? As inferências são a resposta. In TEBEROSKY, A. et al. (Orgs.), Compreensão de leitura: a lingua como procedimento. Porto Alegre: Artmed, p.139-153, 2003.

YUILL, Nicola; OAKHILL, Jane. Children's problems in text comprehension: an experimental investigation. Cambridge: Cambridge University Press, 1991.

Recebido em 08/02/2017

Aceito em 01/08/2017

Publicado em 14/08/2017 